\title{
Initial Analysis of SAR From a Cell Phone Inside a Vehicle by Numerical Computation
}

\author{
Gabriel Anzaldi, Ferran Silva, Member, IEEE, Mireya Fernández, Member, IEEE, Marcos Quílez, Member, IEEE,
} and Pere J. Riu*, Senior Member, IEEE

\begin{abstract}
The purpose of this paper is to analyze the influence of the metallic structures of a realistic car body frame on the specific absorption rate (SAR) produced by a cell phone when a complete human body model is placed at different locations inside the vehicle, and to identify the relevant parameters responsible for these changes. The modeling and analysis of the whole system was conducted by means of computer simulations based on the full wave finite-difference time-domain (FDTD) numerical method. The excitation considered was an $835 \mathrm{MHz} \lambda / 2$ dipole located as a handsfree communication device or as a hand-held portable system. We compared the SAR at different planes on the human model, placed inside the vehicle with respect to the free space situation. The presence of the car body frame significantly changes the SAR distributions, especially when the dipole is far from the body. Although the results are not conclusive on this point, this change in SAR distribution is not likely to produce an increase above the limits in current guidelines for partial body exposure, but may be significant for whole-body exposure. The most relevant change found was the change in the impedance of the dipole, affecting the radiated power. A complementary result from the electromagnetic computations performed is the change in the electromagnetic field distribution inside a vehicle when human bodies are present. The whole vehicle model has been optimized to provide accurate results for sources placed inside the vehicle, while keeping low requirements for computer storage and simulation time.
\end{abstract}

Index Terms-Automotive simulation, computational electromagnetics, EMI modeling, FDTD, SAR.

\section{INTRODUCTION}

$\mathbf{T}$ HE USE of cell phones has increased exponentially in the recent years and has become a ubiquitous element in daily life. These devices produce a non-negligible amount of electromagnetic energy in the radiofrequency band, and this fact has raised public concern about the possible hazards associated to the electromagnetic radiation.

Manuscript received July 7, 2005; revised August 5, 2006. This work was supported in part by the Spanish Ministerio de Educación y Ciencia under Grant DPI2001-0897-C02-01 and GrantDPI2004-07865-C02-01. Asterisk indicates corresponding author.

G. Anzaldi was with the Department of Electronic Engineering, Universitat Politècnica de Catalunya (UPC), Barcelona 08034, Spain. He is now with Dielro, Barcelona 08790, Spain.

F. Silva and M. Fernández are with the Electromagnetic Compatibility Group, Department of Electronic Engineering, Universitat Politècnica de Catalunya, Barcelona 08034, Spain

M. Quílez is with the Department of Electronic Engineering, Universitat Politècnica de Catalunya, Barcelona 08034, Spain.

*P. J. Riu is with the Electromagnetic Compatibility Group, Department of Electronic Engineering, Universitat Politècnica de Catalunya, Barcelona 08034, Spain (e-mail: priu@eel.upc.edu).

Digital Object Identifier 10.1109/TBME.2006.889776
The effects on human health considered by exposure guidelines at cell phone frequencies of operation arise from the heating produced by currents induced by electromagnetic fields on media with finite conductivity, such as human tissues [1]. The basic dosimetric unit used to evaluate this kind of exposure for frequencies below $10 \mathrm{GHz}$ is the power deposited by unit mass [specific absorption rate (SAR)] [2], [3].

Standards that define SAR assessment procedures for personal communication systems are mostly concerned with the certification of the devices [4]-[6] and rely primarily on experimental measurements using actual phones and head phantoms. Current efforts using numerical methods are aimed to define human head models, phone models, and other computation details that allow the comparison between different numerical approaches and between numerical and experimental procedures [23]. Thus, the computational domains used try to replicate the environment defined in the standards. However, actual exposure in real life is not always produced in a free-space like situation.

Partially closed environments, like transportation vehicles, are especially harsh scenarios for several reasons: the metallic structures produce large reflections; the structure itself is large compared to the wavelength, but near field effects must be considered; and the reflective surfaces are mostly curved. These scenarios could be characterized by measurements, and although the procedure can be very complex some successful attempts have been made [18]. Numerical computation methods constitute an attractive alternative way for those scenarios. Different numerical techniques are used to estimate SAR in the human body, and particularly the finite-difference time-domain (FDTD) method has been widely employed in recent decades [7]. Although some approaches to evaluate the effects on SAR arising from reflecting walls located near to a head model have been made [8], modeling a whole vehicle with enough spatial resolution to include a realistic human body results in a computationally large problem which is still unsolved. This problem must be attacked using a rigorous computation method, like FDTD, instead of simpler methods based on plane wave propagation, but the size of the scenario compared to the wavelength and the need to model fine grain details makes it computationally challenging [19]. Methods like those described in [24] involve practical details like rotating the head model to maintain the cell phone model oriented along the computation axis, to be able to obtain meaningful results. Increasing the size of such models to include a passenger vehicle would require an amount of storage and processing time only available in a few top today's supercomputers.

To finally assess the exposure in the described environments requires an iterative process to approximate the modeling 


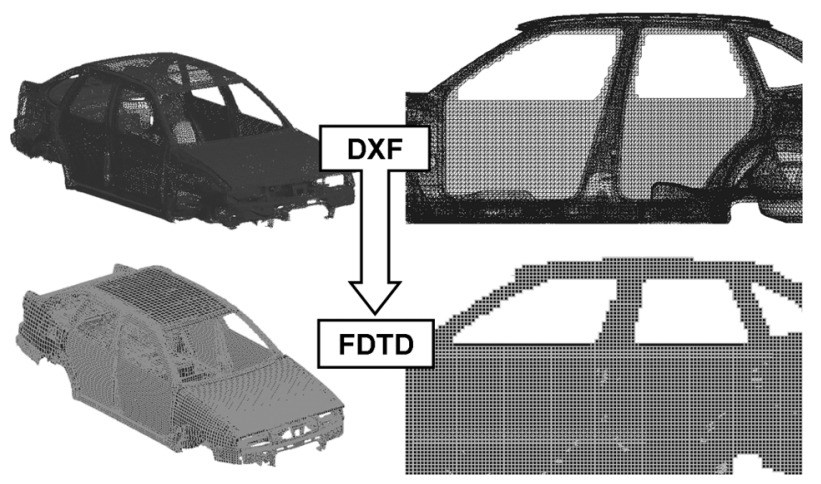

Fig. 1. Original mechanical CAD data in DXF format and final FDTD model.

requirements described in [23], [24] with those arising from the presence of the environment. The methods and results we present in this paper are the first step in such a process and provide some insight into the expected changes on power deposition for a particular environment that will help in directing subsequent approximation steps.

We present an approach that enables us to include a complete human body model with different tissue types and reasonable resolution, placed inside a realistic car body frame model. The approximation taken is based on the separate validation of both; the electromagnetic calculations inside the empty car frame, performed in previous works; and the computation of SAR produced in the near field, which is presented in Section III.

The car model was obtained from the manufacturer (SEAT SA, Barcelona, Spain) as a mechanical CAD file in DXF format. Mechanical details smaller than the FDTD cell size used in the calculations were removed before the simulation mesh was built. Despite this, an additional cleaning process was required. Fig. 1 shows the car body frame model in its original form and the final mesh used in the calculations. Details on the whole process and final parameters used can be found in [10].

There are no publications presenting results of field distribution inside a vehicle using a model of resolution comparable to the one used here, so calculations in the empty car frame were compared to experimental measurements. The estimated uncertainty in the measurements was used as a criterion for the agreement between both. Two kinds of excitations were used, a resonant dipole and a long wire, and measurements of the field distribution and of the voltage coupled to a line were performed at $100 \mathrm{MHz}$ and $1.8 \mathrm{GHz}$. The results obtained and the uncertainty assessment performed are thoroughly described in [16], [17] and will be briefly commented in Section III.

The validation of the SAR calculation was performed by comparison with published work. Because the resolution used in our model is moderate $(3 \mathrm{~mm})$ and we are most interested in assessing changes in the SAR distribution rather than in obtaining a figure comparable with the standards, we used a relatively old work for the comparison [13] instead of more recent and, possibly, more accurate works [24] that only provide numerical values for maximum averaged SAR values.

\section{Human AND Vehicle Models}

There are several procedures for obtaining a realistic body model for SAR calculation purposes. Most of them are based on

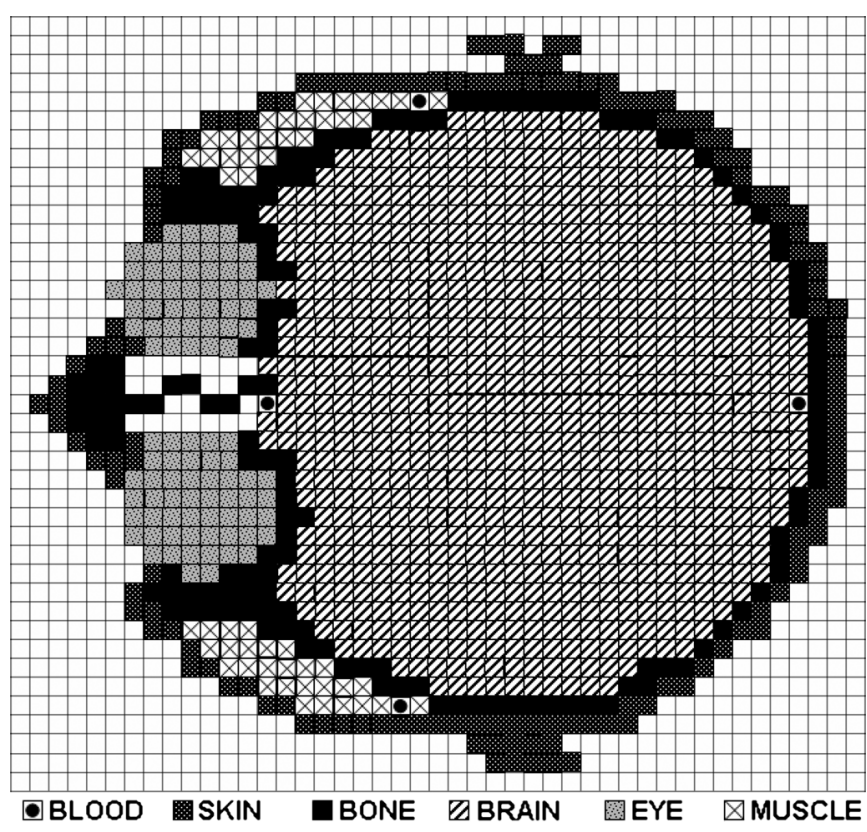

Fig. 2. Meshed cross section of the human model $(\Delta=3 \mathrm{~mm})$ corresponding to plane "front 3" in Fig. 5.

TABLE I

Summary of Dielectric Properties For the Main Tissue Types Used

\begin{tabular}{llcc}
\hline \hline \multirow{2}{*}{ Num } & \multirow{2}{*}{ Tissue } & \multicolumn{2}{c}{ Dielectric parameters } \\
\cline { 3 - 4 } & & $\sigma(\mathrm{S} / \mathrm{m})$ & $\varepsilon$ \\
\hline 1 & Muscle & 1.14 & 53 \\
2 & Bone & 0.04 & 5.4 \\
3 & Brain & 1.46 & 51 \\
4 & Skin & 0.63 & 23 \\
5 & Eye & 1.04 & 59 \\
6 & Heart & 1.196 & 60.34 \\
7 & Kidney & 1.355 & 59.23 \\
8 & Liver & 0.8282 & 47.15 \\
9 & Lungs & 0.4457 & 22.13 \\
10 & Stomach & 1.159 & 65.25 \\
11 & Intestine & 2.131 & 59.95 \\
\hline \hline
\end{tabular}

CT or MRI imaging [12]. There are also commercially available (e.g., Remcom, Inc.) meshes for the human head and even for the whole body. It is also technically possible to create a mesh from freely available slices of a human body (i.e., The Visible Human Project). Instead, we created our own model based on data in the public domain, which allows for a simple scaling and repositioning using any 3-D modeling software.

An adult human body was modeled in a sitting position. Most geometric models of the human body and the organs were obtained from [20] in DXF format and some others were modeled from scratch. After assembling, resizing and coordinating with the car model, the human body was meshed. Fig. 2 shows the cross section, after meshing, corresponding to plane Front 3 in Fig. 5. Table I summarizes the relative permittivity $(\varepsilon)$ and the conductivity $(\sigma)$ at $835 \mathrm{MHz}$ for the main tissue types used [13], [14]. 
The overall computational domain, which includes the vehicle, the human body and the surrounding air, has a volume of $4644 \times 2160 \times 1764 \mathrm{~mm}^{3}$. Uniform cubic meshes with cell sides $(\Delta)$ of $36 \mathrm{~mm}$ were used over the coarse region, outside the vehicle. An intersection of submeshes was defined over the metallic structures, achieving a resolution of $9 \mathrm{~mm}$, and over the human region, achieving a final resolution $\Delta=3 \mathrm{~mm}$. Surrounding these finer zones, transition regions were defined until the coarse area was reached, in order to minimize numerical reflections [11]. Two different views of the final assembled system can be found in the Results section. The use of submeshing in the particular FDTD code forced us to use first-order mur absorbing boundary conditions (ABCs) instead of perfectly matched layers (PMLs) to truncate the simulation space.

SAR calculation was performed using a steady-state sinusoidal waveform at $835 \mathrm{MHz}$. The antenna was modeled as a resonant dipole fed with a voltage source including a $120 \Omega$ series impedance, chosen to have a radiated power (RP) of $0.58 \mathrm{~W}$ with $\Delta=3 \mathrm{~mm}$, and to reduce the number of time steps needed for convergence [15]. Feeding voltage and source impedance were kept constant for all the situations. An accurate model of a cellular phone was not considered and the dipole was placed in vertical position aligned with the FDTD grid ( $z$-axis).

Two setups were used for comparison purposes: the human body in free space and the human-vehicle system. These situations were run for 10000 and 20000 time steps respectively, to reach the steady state condition, taking 10 and $20 \mathrm{hr}$., respectively. The presence of the vehicle body frame increased at least twice the number of time steps needed to reach the steady state condition. The total memory required was 1791 Mbytes for the largest model. The FDTD code is a free distribution called LC (Cray Research, Inc.) [22] running in a Linux box with two Pentium-Xeon 1.8-GHz processors with a sustained performance of 300 MFlops.

\section{VALIDATION}

It is rather difficult to compare the calculation results with experimental measurements in a real car scenario for the electromagnetic field distribution only, let alone when a human body is incorporated. For that reason we decided to validate the calculations of electromagnetic fields in an empty vehicle, as well as the procedure to obtain the SAR in a human body in two different steps.

\section{A. Validation of the Electromagnetic Field Distribution Computations}

Three different situations were modeled and actually built to validate the results for the electromagnetic field distribution inside the car frame. The three situations are briefly described below:

The first one consisted in the characterization of the electromagnetic environment produced by an internal dipole source at $1800 \mathrm{MHz}$. The distribution for the internal field in several planes was obtained and compared to measurements at several discrete points. The measurements were performed using a HI-6005 isotropic broadband E field probe (Holaday Inc.). Differences between calculations and measurements ranging from

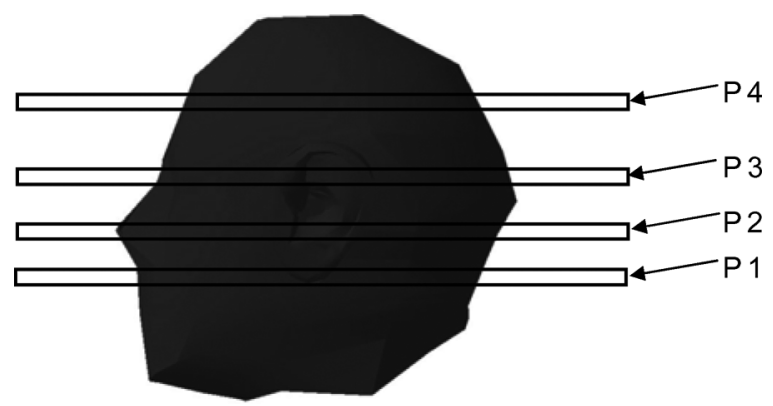

Fig. 3. Head model used for the validation of the SAR calculation procedure.

5\% to $9 \%$ were found. A complete description of the model and experimental setup can be found in [16], [17].

The second setup consisted of a single long copper wire (thin wire structure) radiating at $100 \mathrm{MHz}$ placed inside the test prototype [17]. A grid of nine measurement points was established. The results showed differences ranging from $7 \%$ to $13 \%$.

The third setup was used to calculate the coupling between the electromagnetic fields produced by a wire to another transmission line. Voltage probes were modeled at the end of each wire. The differences between calculations and measurements were reduced to $3 \%$ [10].

The agreement between calculations and measurements was considered satisfactory because the differences were smaller than the estimated uncertainty in the measurements. A more comprehensive explanation of the validation procedure and uncertainty assessment can be found in [17].

\section{B. Validation of the SAR Computation}

To compute the SAR in an FDTD cell $(x, y, z)$, assuming isotropic materials, the following relationship was employed:

$$
\mathrm{SAR}=\frac{|E(x, y, z)|^{2} \sigma(x, y, z)}{d(x, y, z)}
$$

where $\sigma$ is the material conductivity $(\mathrm{S} / \mathrm{m}), E$ is the electric field magnitude (V/m, RMS), and $d$ is the mass density (assumed $\left.1000 \mathrm{~kg} / \mathrm{m}^{3}\right)$.

Computation and postprocessing of SAR data were performed in Matlab (The Mathworks, Inc.), by using the electric fields provided by the FDTD code and the geometry and conductivity information from the mesh files. The 6-field procedure was employed [21] and most of the procedure was manually performed.

We created a simulation model similar to that described in [13]. In this paper, the SAR produced by a cell phone in the human head is reported for several transversal planes, like those shown in Fig. 3. This particular work was chosen because of the similarities in the geometric head model used, the cell sizes used in the discretization, the excitation setup and the fact they provide SAR distributions for some planes.

The human head was modeled using 6 different tissues: muscle, bone, blood, brain, skin and eyes. The cell phone was modeled as a dipole fed by a sinusoidal voltage source incorporating a series resistance selected to obtain a radiated power of $600 \mathrm{~mW}$. The distance between the dipole and the 
TABLE II

COMParison of SAR VALUes USED For the Validation PRocedure (SEe TEXT FOR DETAILS)

\begin{tabular}{ccc}
\hline \hline Plane & Peak SAR in [13] & Peak of SAR \\
\hline 1 & $0.5 \mathrm{~W} / \mathrm{kg}$ & $0.5 \mathrm{~W} / \mathrm{kg}$ \\
2 & $1.3 \mathrm{~W} / \mathrm{kg}$ & $1.3 \mathrm{~W} / \mathrm{kg}$ \\
3 & $1.4 \mathrm{~W} / \mathrm{kg}$ & $1.4 \mathrm{~W} / \mathrm{kg}$ \\
4 & $0.5 \mathrm{~W} / \mathrm{kg}$ & $0.5 \mathrm{~W} / \mathrm{kg}$ \\
\hline \hline
\end{tabular}

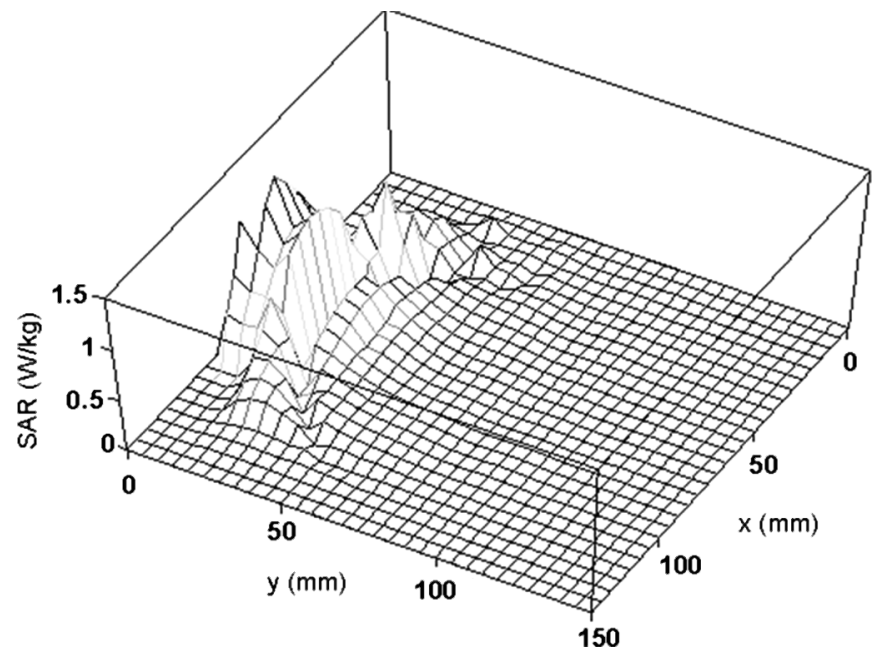

Fig. 4. Peak SAR distribution for plane 3 obtained with the methodology described in the text $(\Delta=5 \mathrm{~mm})$. It can be directly compared with Fig. 7 in [13].

closest part of the head was $2 \mathrm{~cm}$. The dipole was placed in vertical position aligned with the FDTD grid.

Table II reports the maximum nonaveraged values found in each of the planes depicted in Fig. 3, showing our calculations and the corresponding values obtained in [13]. Because there is limited information about numerical values in [13], values in Table II are obtained from figures in this work. The uncertainty associated with this method corresponds to the resolution of the plots in [13], so all values in Table II are truncated to a resolution of $0.1 \mathrm{~W} / \mathrm{kg}$. Fig. 4 shows the SAR distribution for plane 3 in Fig. 3.

\section{RESULTS}

The two situations described in Section II were studied for two different emitter locations. The first one represents a handsfree communication setup, with the phone placed to the right of the steering wheel at $d=228 \mathrm{~mm}$ from the driver's right hand (Fig. 6). The second case represents the front passenger using a hand-held mobile phone. The hand-held communication device was situated at several distances from the head. Results for the distances $\mathrm{d}=5.1 \mathrm{~cm}$ and $\mathrm{d}=9 \mathrm{~mm}$ are presented (Fig. 10). In all the situations, the dipole was in vertical position, aligned with the FDTD grid ( $z$-axis), so the positions do not correspond

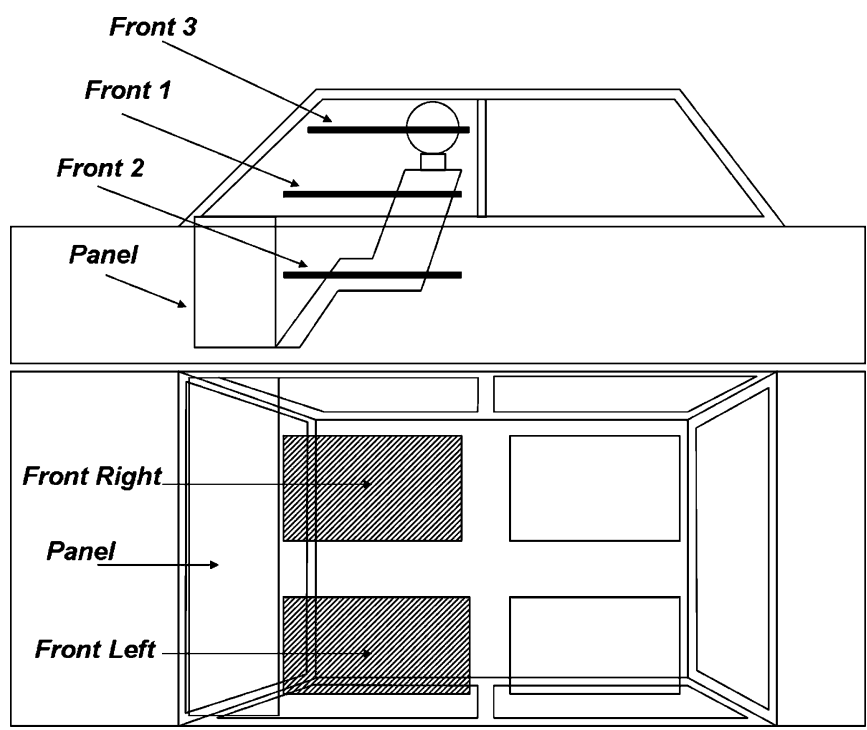

Fig. 5. Schematic description of the evaluated regions.

TABLE III

Net Power And Dipole Current at Feed Point for DifFerent SituATIONS AND Distances From Dipole to HEAD

\begin{tabular}{lcccc}
\hline & \multicolumn{2}{c}{$\mathrm{d}=9 \mathrm{~mm}(3 \mathrm{cells})$} & \multicolumn{2}{c}{$\mathrm{d}=5.1 \mathrm{~cm}(17$ cells $)$} \\
\cline { 2 - 5 } & $\begin{array}{c}\text { Feed current } \\
\text { Power }\end{array}$ & $\begin{array}{c}\text { Mag. (Phase) } \\
{[\mathrm{mW}]}\end{array}$ & $\begin{array}{c}\text { Power } \\
{[\mathrm{mA}][\mathrm{deg}]}\end{array}$ & $\begin{array}{c}\text { Feed current } \\
\text { Mag. (Phase) } \\
{[\mathrm{mA}][\mathrm{deg}]}\end{array}$ \\
\hline Scenario & 580 & 124 & 580 & 124 \\
Dipole & $544(-6 \%)$ & $138\left(12^{\circ}\right)$ & $567(-2 \%)$ & $137\left(5^{\circ}\right)$ \\
$\begin{array}{l}\text { Dipole + body } \\
\text { vehicle }+\end{array}$ & $415(-28 \%)$ & $109\left(32^{\circ}\right)$ & $415(-28 \%)$ & $109\left(32^{\circ}\right)$ \\
$\begin{array}{l}\text { Dipole + body } \\
+ \text { vehicle }\end{array}$ & $387(-33 \%)$ & $118\left(34^{\circ}\right)$ & $403(-30 \%)$ & $117\left(33^{\circ}\right)$ \\
\hline \hline
\end{tabular}

to those described in the standards for compliance assessment [4].

The SAR values were calculated over three planes for the driver and the front passenger's location, as depicted in Fig. 5; crossing the model of the human body at the heart (Plane 1); thighs (Plane 2), and eyes (Plane 3). Only the results significant to the objective of this paper are presented.

It is known that when an antenna is placed near a dissipative object, changes in the radiated power are produced because the antenna impedance changes. In the present case, when the antenna is introduced inside a vehicle, even larger changes are produced. It is a usual practice to normalize the SAR results either to the net radiated power or to the current in the feed gap [23], thus allowing for comparison of results. Whether this is appropriate in this work will be discussed in the next section. The results presented correspond to a constant feeding voltage and source output impedance. Table III summarizes some of the values for the net power, computed from the voltage and current in the feed, and current at the feed gap for different situations. The value for the impedance in free space is assumed to be $75 \Omega$, thus, all other values are referenced to this one. 


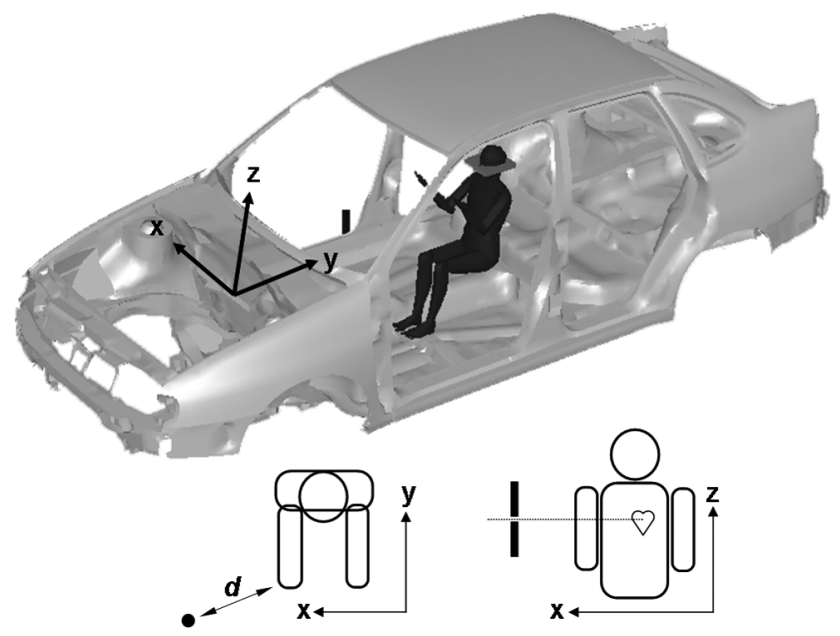

Fig. 6. Relative positions of human model and cell phone inside the vehicle for the hands-free case. Plane 3 (eyes) is shown (doors and covers removed for visualization purposes). Schematic projections in planes $X Y$ and $X Z$ are provided (not to scale) to depict the relative position of the dipole with respect to the body. $d=338 \mathrm{~mm}$.

\section{A. Emitter as a Hands-Free Device}

Fig. 6 shows the relative position of the human body model within the vehicle and the cell phone. Plane 3 (eyes) is depicted. Doors have been removed from the car model in order to allow the interior view

1) Case A1. Driver and Hands-Free Device, Plane 1 (Heart) Front Left: Fig. 7(a) shows the SAR distribution for plane 1 (driver's thorax and arms) for the human body in free space. The highest values of SAR are found in the right arm (closest to the phone) with a value of $58 \mathrm{~mW} / \mathrm{kg}$. SAR distribution within the arm is almost constant because of its proximity to the source. Penetration of the field in the thorax is very poor, with maximum SAR values ranging from $10 \mathrm{~mW} / \mathrm{kg}$ to a peak of $18 \mathrm{~mW} / \mathrm{kg}$.

Significant changes are observed when the car body frame is introduced [Fig. 7(b)], mainly in the thorax region over the back left lateral side, where the SAR grows, reaching a new local peak of $10 \mathrm{~mW} / \mathrm{kg}$. The regions with significant SAR values in freespace experienced a small increase, while the arms experienced a small decrease. The highest value was in the same position, but experienced a $4 \%$ decrease.

2) Case A2. Driver and Hands-Free Device, Plane 3 (Eyes) Front Left: The results obtained for the human body in free space are displayed in Fig. 8(a). A peak SAR of $1.7 \mathrm{~mW} / \mathrm{kg}$ on the right side of the forehead is observed, with a decrease towards the interior of the head. When the vehicle is introduced, Fig. 8(b), there is an increase over the whole region, and, in addition, the peak SAR changes its position from front to back. The value of that new peak is about $5.4 \mathrm{~mW} / \mathrm{kg}$.

3) Case A3. Driver and Hands-Free Device, Plane 2 (Thighs) Front Left: The maximum value reached in free space is 3.9 $\mathrm{mW} / \mathrm{kg}$, on the thigh closest to the dipole, Fig. 9(a). This peak value increases to $11.3 \mathrm{~mW} / \mathrm{kg}$ for the human-vehicle system and changes its position from the right knee to the right side of the right leg as shown in Fig. 9(b). The SAR values in the other areas showed a general increase, mainly in the areas far from
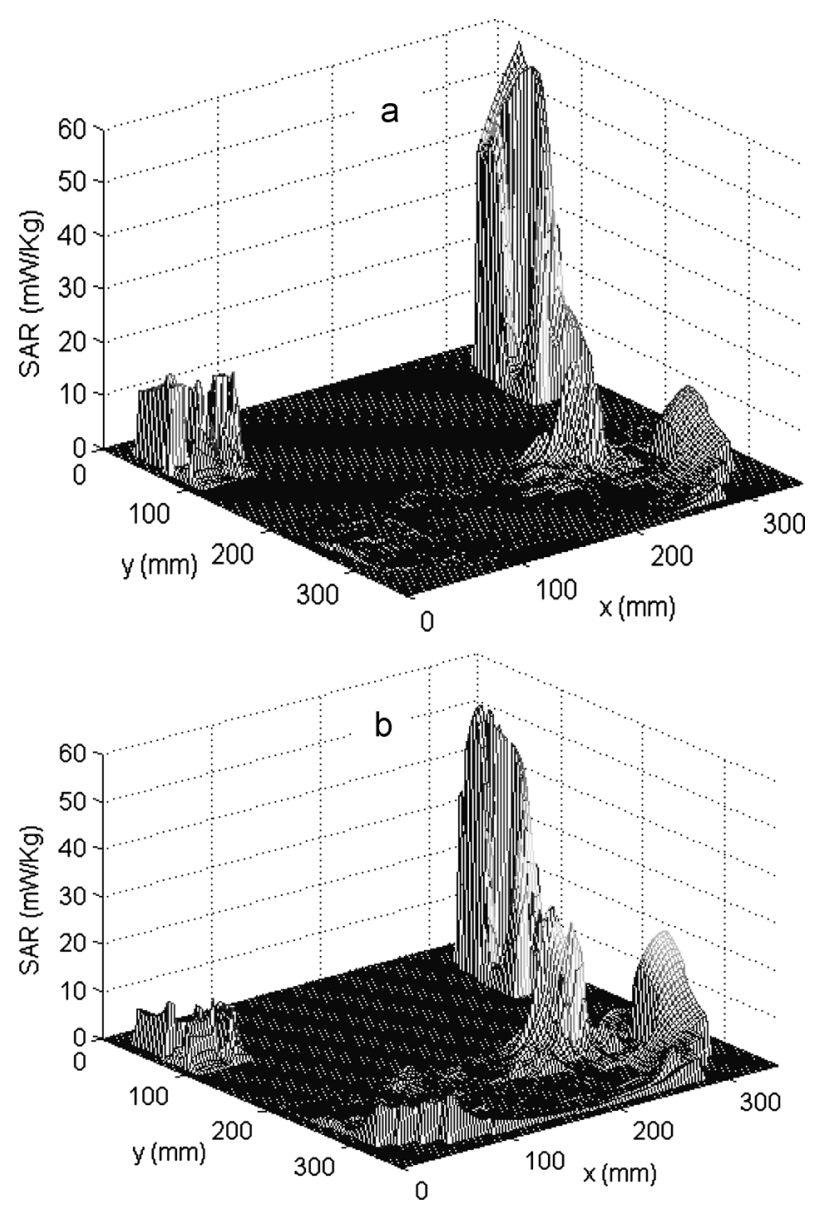

Fig. 7. Peak SAR values for the hands-free case, corresponding to case A1. Plane 1 (heart) is displayed. (a) Human body in free space. (b) Human model inside the vehicle.

the dipole. Values of SAR in the middle of the legs are very low, because of the low conductivity of bone tissue.

\section{B. Emitter as a Hand Held Device}

Fig. 10 shows the relative positions of the emitter and the passenger, simulating a cell phone used as a hand-held device. Plane 3 (eyes) is depicted.

1) Case B1.1. Front Passenger Hand-Held Device at $5.1 \mathrm{~cm}$ From the Right Ear. Plane 3 (Eyes) Front Right: Fig. 11(a) and (b) depicts the SAR distribution for plane 3 (eyes) when the passenger uses the cellular phone hand held at $5.1 \mathrm{~cm}$ from the head, in free space and inside the vehicle respectively. The peak of SAR for the passenger in free space is $0.27 \mathrm{~W} / \mathrm{kg}$, located on the area closest to the dipole, and $15 \mathrm{~mm}$ toward the interior of the head. In the interior of the head, SAR values diminish to the point of being too small to be evaluated. For the body model inside the vehicle the peak SAR maintains its position but decreases by $8 \%$. In the central part of the head there is a light increase of about $10 \%$, being larger in the area opposite to the dipole $(80 \%)$.

2) Case B1.2. Front Passenger Hand Held Device at $9 \mathrm{~mm}$ From the Right Ear. Plane 3 (Eyes) Front Right: When the dipole is closer to the head the differences between the free- 

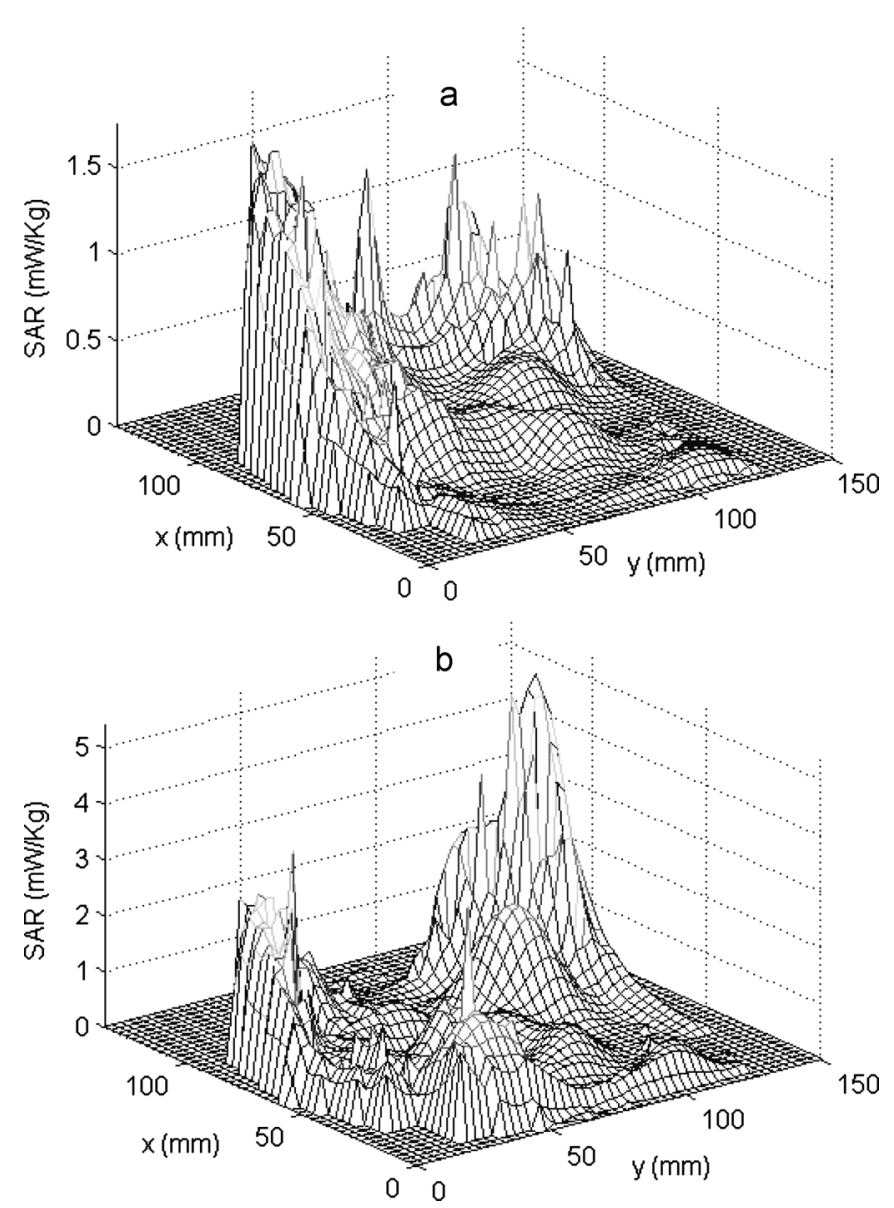

Fig. 8. Peak SAR values for the hands-free case, corresponding to case A2. Plane 3 (eyes) is displayed. (a) Human body in free space. (b) Human model inside the vehicle.

space situation, Fig. 12(a), and the body inside the vehicle situation, Fig. 12(b), are smaller than $1 \%$. Peak value for the SAR is about $2 \mathrm{~W} / \mathrm{kg}$. It is worth noticing that this is not an averaged value over $10 \mathrm{~g}$ of tissue. This value corresponds to a simulation cell of $27 \mathrm{~mm}^{3}$ in volume, i.e., $27 \mathrm{mg}$, thus, it cannot be used to directly compare with limits in the standards.

3) Case B2. Front Passenger Hand Held Device at $9 \mathrm{~mm}$ From the Right Ear. Plane 1 (Heart) Front Right: The differences in SAR distribution for the planes located far from the dipole are again large. In free space, Fig. 13(a), the maximum value of SAR is $3.8 \mathrm{~mW} / \mathrm{kg}$, and is located on the right part of the thorax. High values of SAR are also observed in the right arm, while values are very low in the left part of the body. Fig. 13(b) shows the values of SAR calculated when the body is in the interior of the vehicle. The maximum SAR value increases to $6.6 \mathrm{~mW} / \mathrm{kg}$, located in the same place as in the free space situation. In the left region, a peak of $1.5 \mathrm{~mW} / \mathrm{kg}$ appears in a zone that displayed null values for the free space situation. An SAR increase is also found in the right arm, while the left arm experiences a decrease.

4) Case B3. Front Passenger Hand Held Device at $9 \mathrm{~mm}$ From the Right Ear. Plane 2 (Thighs) Front Right: The maximum SAR value found in plane 2 for free space corresponds
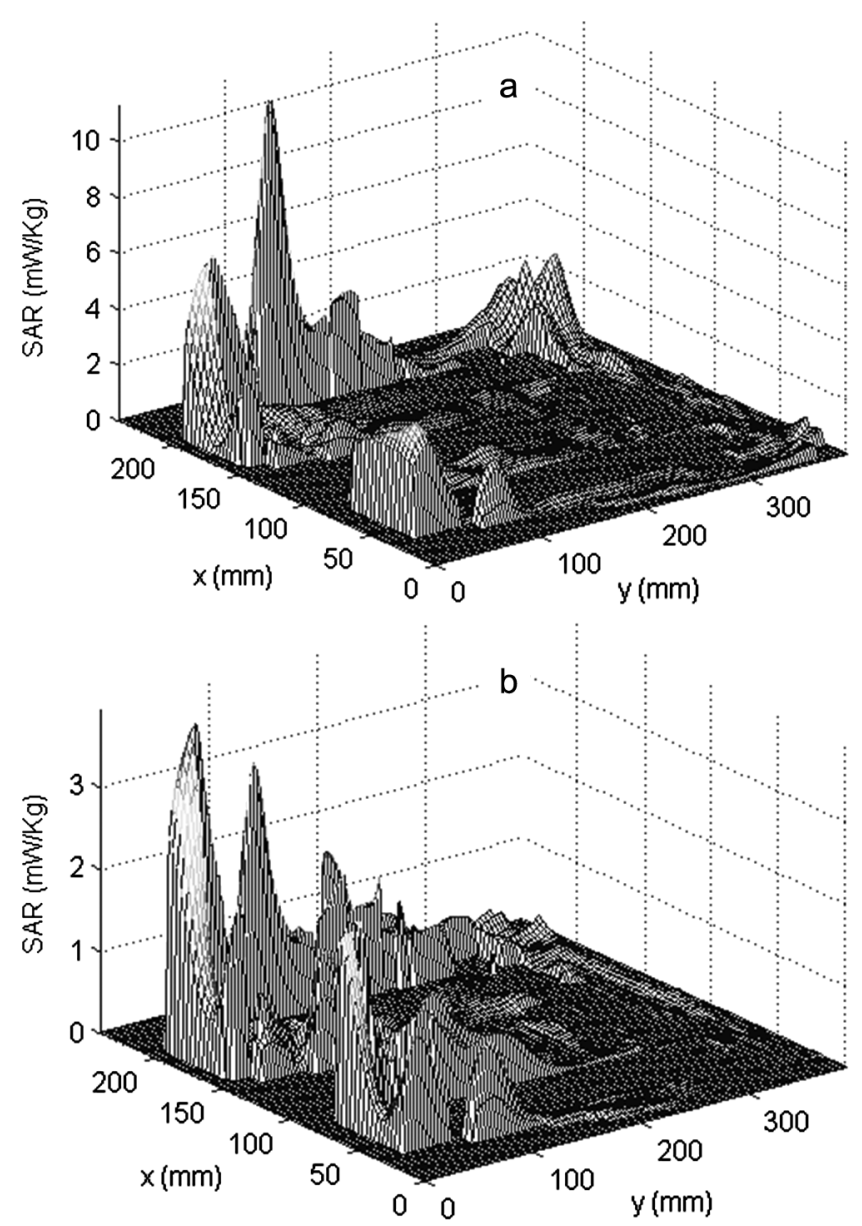

Fig. 9. Peak SAR values for the hands-free case, corresponding to case A3. Plane 2 (thighs) is displayed. (a) Human body in free space. (b) Human body model inside the vehicle.

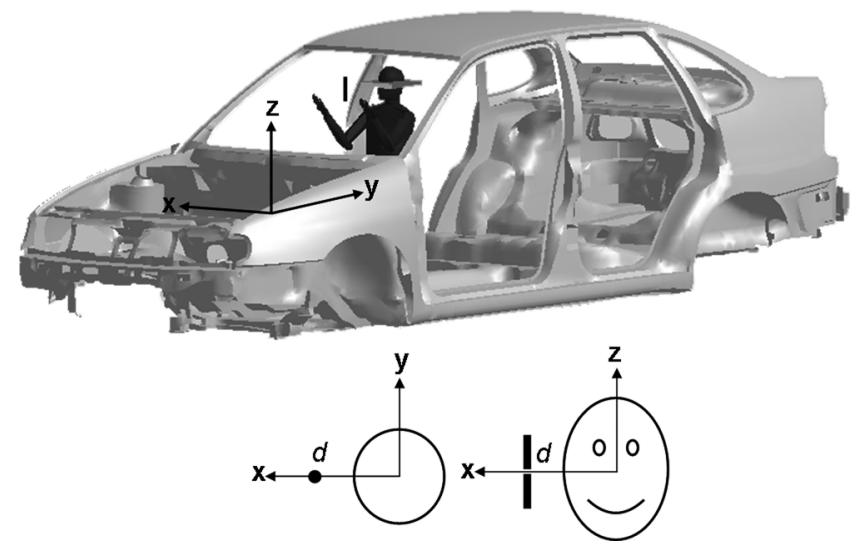

Fig. 10. Relative positions of human model and cell phone inside the vehicle for the hand-held case. Plane 3 (eyes) front right is shown. Schematic projections in planes $X Y$ and $X Z$ are provided (not to scale) to depict the relative position of the dipole with respect to the head. $d=9 \mathrm{~mm}, 51 \mathrm{~mm}$.

to the thigh closer to the dipole, displaying values of about 1.6 $\mathrm{mW} / \mathrm{kg}$ [Fig. 14(a)]. When the car frame is introduced, there is a small decrease in that maximum but the values in the areas opposite to the dipole show a significant increase with local maximums of about $0.5 \mathrm{~mW} / \mathrm{kg}$ [Fig. 14(b)]. 

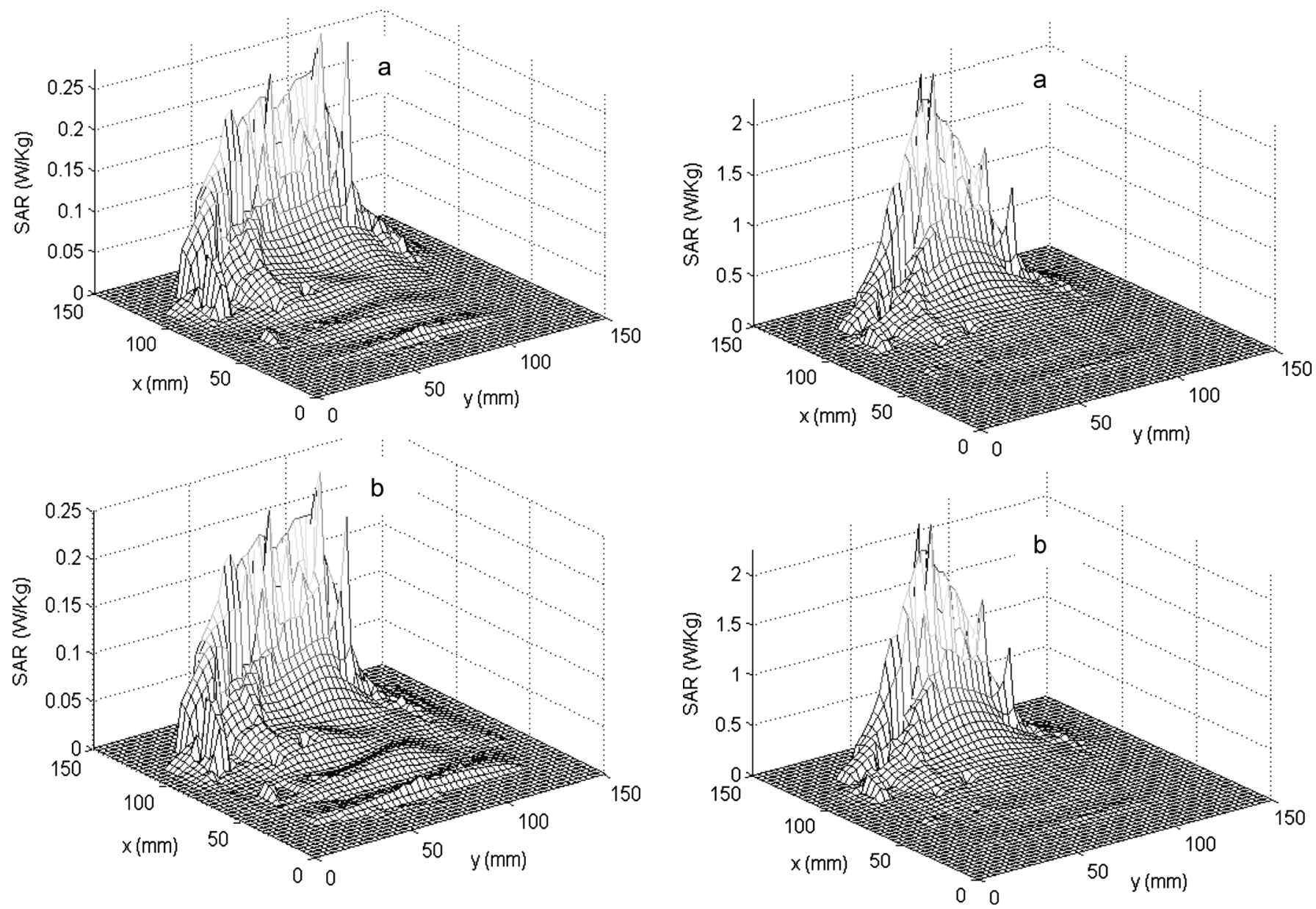

Fig. 11. Peak SAR values for the hand-held case, corresponding to case B1.1 $(\mathrm{d}=5.1 \mathrm{~cm})$. Plane 3 (eyes) is displayed. (a) Human body in free space. (b) Human body model inside the vehicle.

\section{DISCUSSION}

Considerable effort is currently being made in numerical dosimetry to find repetitive and consistent results between different head models, phone positions, numerical method implementations, etc.; and to be able to compare these results to experimental values found using SAM [24]. This task requires human models of about $1 \mathrm{~mm}$ resolution, accurate modeling of the cell phone and a very precise description of phone position. It was not the aim of our work to add to this topic, chiefly because using such detailed models along with a body car frame requires computational resources beyond our possibilities. In addition, mixing the problem of the consistency of results among anatomically correct models and the introduction of a realistic partially closed environment would have produced a set of results difficult to interpret. We have taken some decisions about models, complexity, results presented, etc. that certainly have some impact on the interpretation of the results:

1) Phone Model and Position: There is a defined "standard" phone for testing purposes which is often used in simulation. Instead, for the sake of simplicity we have used a dipole. Some differences in SAR deposition will arise from that fact. In addition, the phone model was not in the standard positions described in standards, because doing this generally implies per-

Fig. 12. Peak SAR values for the hand-held case, corresponding to case B1.2 $(\mathrm{d}=9 \mathrm{~mm})$. Plane 3 (eyes) is displayed. (a) Human body in free space. (b) Human body inside the vehicle.

forming a rotation of the head model to keep the phone aligned with the FDTD grid [23] as discussed in the introduction.

2) Radiated Power: We used a constant voltage to feed the dipole. It is usual to normalize results to net radiated power or current feed. It is clear from Table III that the reduction in radiated power produced by the car body frame is very high, about $30 \%$, while the reduction in current is only about $10 \%$, and the reduction in calculated SAR is smaller. Whether or not it is appropriate to normalize the results and the appropriate normalization values is discussed later, but it is clear that this will be a key point when considering these scenarios.

3) Data Presented: We are presenting SAR distributions on discrete planes instead of $10 \mathrm{~g}$ averaged values. By doing this we cannot directly compare our results against the standards and possibly some information regarding nonobserved planes could be lost. In addition, nonobserved planes may show behaviors that are in contradiction with the observed ones. For that reason all conclusions about whole-body or local SAR increases or decrements must be taken with much care. However looking at SAR distributions provides more insight than obtaining a single figure. In addition, obtaining a single figure may mask errors in the process. It will certainly be necessary to obtain these figures at a later time, when other issues have been resolved. 

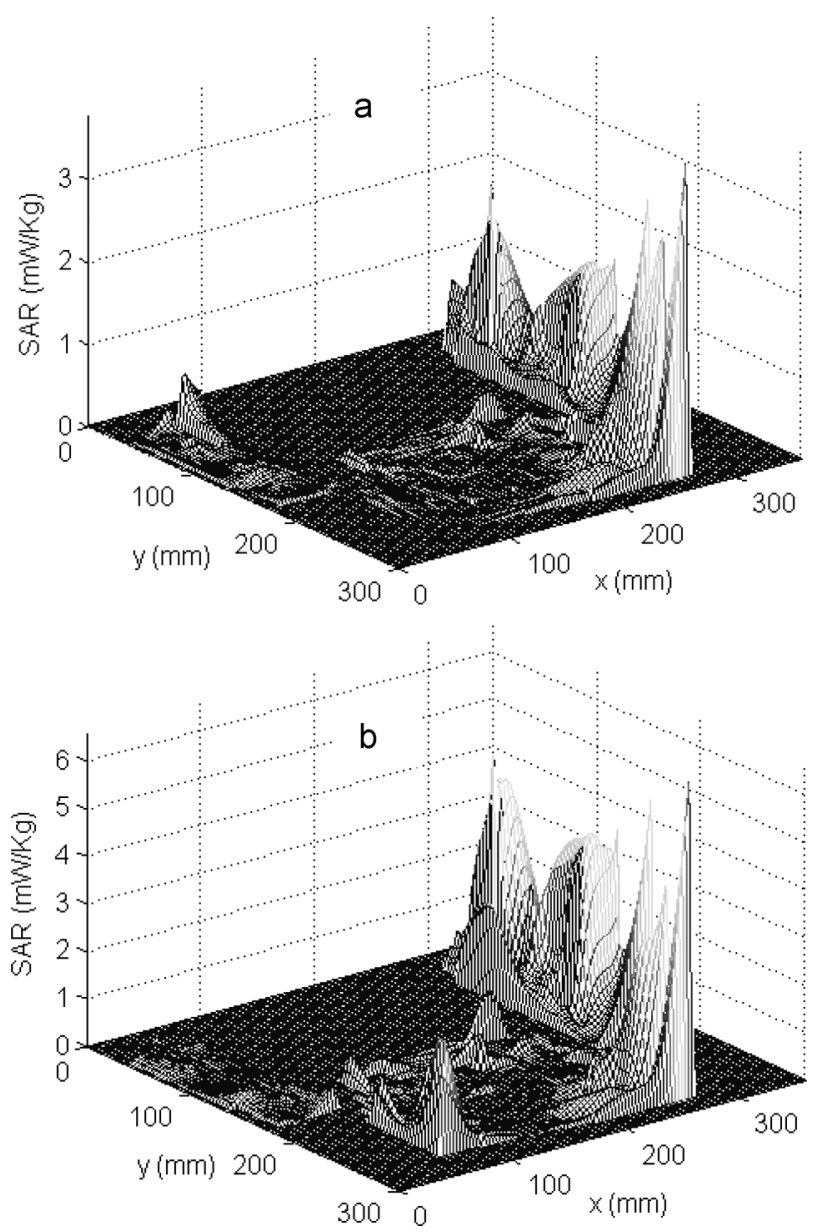

Fig. 13. Peak SAR values for the hand-held case, corresponding to case B2. Plane 1 (heart) is displayed. (a) Human Body in free space. (b) Human body model inside the vehicle.

4) Simulation Parameters: We used ABC boundary conditions instead of PML. In our case, this was forced by the FDTD code used. Although PML is known to produce better results, the problem treated here is much dependent on the reflections by the metallic structures rather than by the boundaries, and we do not expect significant changes produced by that fact. Also the election of the SAR calculation method is not the most accurate, but the differences reported by other authors [21] are small and in the range, for example, of the uncertainties in our measurements (used to verify the calculations).

We have presented a total of 7 different situations, each one for the free-space and the body-in-vehicle setups. There is an almost infinite number of different combinations of the human body position and the excitation (cell phone) location. However the selection presented shows a repetitive pattern, and it seems sensible to expect a similar behavior for other planes, passengers and cell phone positions.

The maximum SAR values in each plane are found in the body part closest to the excitation, for both the free-space setup and the body-in-vehicle setup. The value of the maximum experiences a small decrease, in the order of $5 \%$, when the body is placed inside the car frame. This effect may be caused by a change in the radiated power due to the change in the dipole radiation resistance when the car frame is present. We have main-
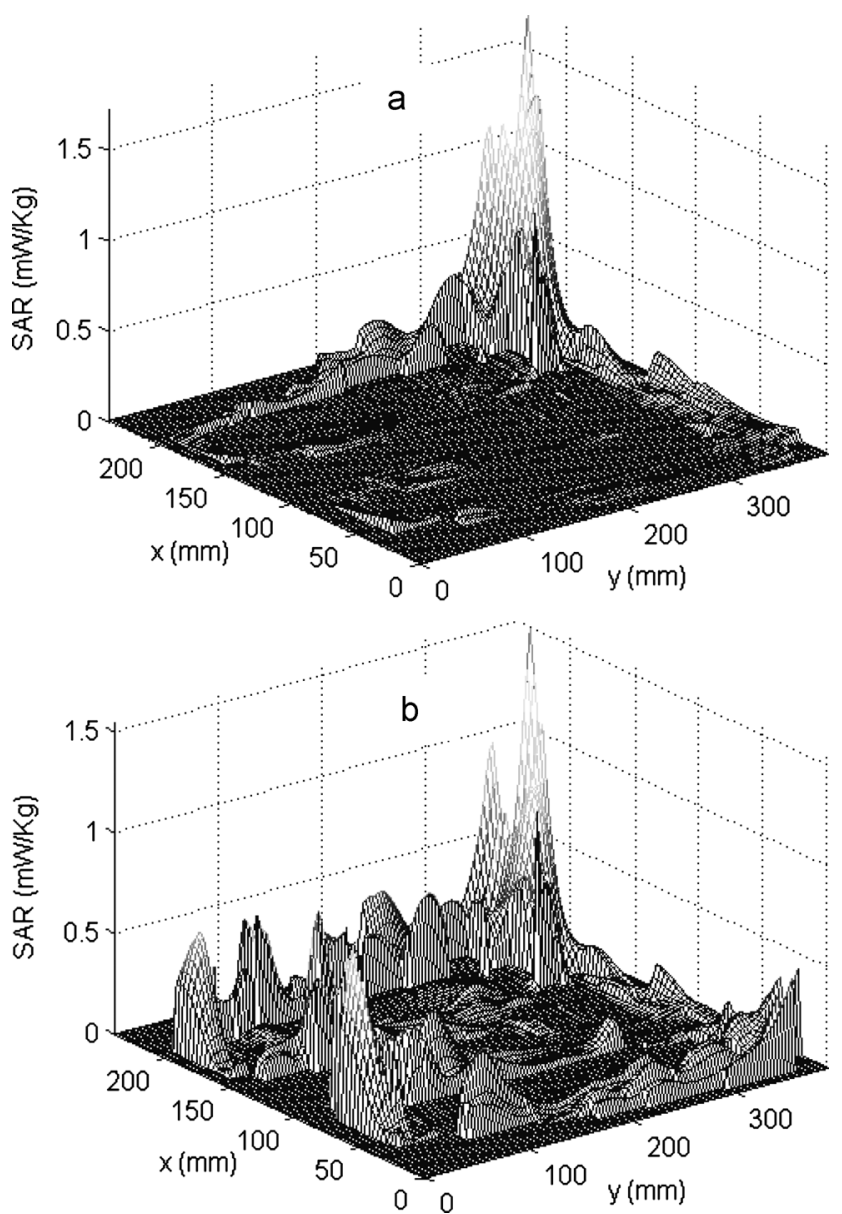

Fig. 14. Peak SAR values for the hand-held case, corresponding to case B3. Plane 2 (thighs) is displayed. (a) Human body in free space. (b) Human body model inside the vehicle.

tained constant the values of the excitation voltage and source impedance. Table III provides values for the net radiated power and feed current for some situations, so it is possible to scale values to these figures. It is difficult to determine the actual behavior of a real phone when mismatch in the antenna impedance is found. Some authors [23] believe that the real SAR value would be between the values provided by scaling to the radiated power and the feed current. This might be true when only the effects of the head for a near phone in free space are considered, but it is no so clear when the antenna is far from the body and inside a semi-closed environment. Further experiments or calculations, possibly in a more simplistic scenario, must be performed to elucidate this point.

There is an increase in the SAR in the regions far from the excitation when the body is placed inside the vehicle. This increase is more apparent for the situations displaying small absolute values of SAR (Cases A2, A3, B2, and B3). It is less apparent when absolute values of SAR are higher (Case A1) and can hardly be observed in the graphs for cases B1.1 and B1.2, although it is present when numerical values are compared. The observed increases in the regions far from the dipole are caused by reflections in the metallic structures of the car frame.

Similar increases in the whole body are likely to encountered, thus, the total averaged SAR will probably increase when a cell 
phone is used inside a vehicle. The peak values found in the planes studied (except B1.1 and B1.2) are always lower than the limit of $80 \mathrm{~mW} / \mathrm{kg}$ for the whole body averaged SAR in the exposure guidelines, but there are regions in the body, not included in this work, that will display higher values of SAR. Thus, the data obtained is not conclusive with respect to the question if the whole body averaged SAR limit can be exceeded when a cell phone is used inside a vehicle, and a more comprehensive study is needed.

The results obtained for cases B1.1 and B1.2 would lead to the conclusion that partial body limits will not be exceeded when using a phone inside a vehicle, provided they are not exceeded in free-space. Other works have found larger changes when reflective walls are placed near the head or the dipole. However, in the case studied here, the closest metallic structure is the roof of the vehicle, because the windows are nonconducting structures. Other situations, like a cell phone worn on the waist may lead to different results and deserve further study.

\section{CONCLUSION}

We show the feasibility of obtaining sensible and accurate results for the electromagnetic field distribution within a complex and large (compared to the wavelength) structure such as a passenger car. A simple FDTD code with second-order accuracy and a low cost computation system are used, and results compared with experimental measurements. We have also shown that SAR calculations out of these field distributions do compare well with the results obtained in other works.

We show the influence of the vehicle body frame on the SAR distributions, when compared with free-space situations, for two realistic scenarios: a driver with a hands-free setup and passenger using a hand-held phone. Inside a vehicle, where multipath propagation, reflections and scattering must be accounted for, the SAR distribution undergoes large changes. However the larger changes are observed in those situations where the absolute values of SAR are quite low compared to those in the international guides limiting human exposure to RF fields for partial body exposure ( 2 or $4 \mathrm{~W} / \mathrm{kg}$ ). For the only situation displaying values comparable to the limits, passenger using a hand-held device, the differences found between free-space and being inside the vehicle are small. In this situation, most of the coupling is in the near field and the most relevant parameters are the distance from the dipole to the head [1] and, not addressed here, the details of the head mesh; and those of the phone and phone position.

Our results suggest that a key factor when dealing with these scenarios is the actual radiated power of the phone, because a large change in the antenna impedance is observed when the metallic structure of the vehicle is considered. More research and a better understanding of the behavior of actual phones is needed to address this point.

For whole body deposition, when the phone is far from the user, our results suggest that using a cell phone inside a vehicle produces an increase of SAR values over the whole body, but this observation must be taken with much care because only few planes are observed. It is clear, however, that the vehicle structure is responsible for large relative changes in SAR in that situation, thus, a standard and realistic vehicle should probably be defined to make progess in that direction. Our results are of comparative nature and are valuable as indicators of the changes expected between the free space situation and other more realistic exposure conditions, but cannot be directly used for compliance assessment.

The results obtained can also be used to gain knowledge about the influence of passengers over the electromagnetic field distribution inside a vehicle, which is of interest in other research areas, such as electromagnetic compatibility, since the number of electromagnetic sources inside passenger vehicles is growing rapidly.

\section{REFERENCES}

[1] P. J. Riu and K. R. Foster, "Heating of tissue models by near-field exposure to a dipole," IEEE Trans. Biomed. Eng., vol. 46, no. 8, pp. 911-917, Aug. 1999.

[2] ICNIRP, "Guidelines for limiting exposure to time-varying electric, magnetic, and electromagnetic fields (up to $300 \mathrm{GHz}$ )," Health Phys., vol. 74, no. 4, pp. 494-522, 1998.

[3] Safety Levels With Respect to Human Exposure to Radio Frequency Electromagnetic Fields, $3 \mathrm{KHz}$ to $300 \mathrm{GHz}$, IEEE Std. C95.1-2005, IEEE, 2006.

[4] Basic Standard for the Measurement of Specific Absorption Rate Related to Human Exposure to Electromagnetic Fields From Mobile Phones (300 MHz-3 GHz), EN 50631:2001, CENELEC, 2001, CENELEC.

[5] IEEE Recommended Practice for Determining the Peak Spatial-Average Specific Absorption Rate (SAR) in the Human Body Due to Wireless Communications Devices: Measurement Techniques, IEEE Std. 1528-2003, Inst. Elect. Electron. Eng., 2003, New York.

[6] Human Exposure to Radio Frequency Fields From Hand-Held and Body-Mounted Wireless Communication Devices-Human Models, Instrumentation, and Procedures-Part 1: Procedure to Determine the Specific Absorption Rate (SAR) for Hand-Held Devices Used in Close Proximity to the Ear (Frequency Range of $300 \mathrm{MHz}$ to $3 \mathrm{GHz}$ ), IEC 62209-1 (2005-02), Int. Electrotech. Commission, 2005, Geneva.

[7] K. S. Yee, "Numerical solution of initial boundary value problems involving Maxwell's equations in isotropic media," IEEE Trans. Antenna. Propag., vol. AP-14, pp. 302-307, 1966.

[8] P. Bernardi, M. Cavagnaro, and S. Pisa, "Evaluation of the SAR distribution in the human head for cellular phones used in a partially closed environment," IEEE Trans. Electromagn. Compat., vol. 38, no. 3, pp. 357-366, Aug. 1996.

[9] A. Taflove and S. Hagness, Computational Electrodynamics, $2 \mathrm{nd}$ ed. Norwood, MA: Artech House, 2000, pp. 109-143.

[10] G. Anzaldi, P. J. Riu, R. Santos, and F. Silva, "Finite difference time domain low cost modelling for automotive environments," in Proc. IEEE Int. Symp. EMC, Santa Clara, CA, Aug. 2004, vol. 3, pp. 775-780.

[11] J. W. Nehrbass and R. Lee, "Optimal finite-difference sub-gridding techniques applied to the Helmholtz equation," IEEE Trans. Microw. Theory Tech., vol. 48, no. 6, pp. 976-984, Jun. 2000.

[12] J. Gambarelli, G. Guerinel, L. Chevrot, and M. Mattei, Computerized Axial Tomography. New York: Springer, 1977.

[13] H. Y. Chen and H. H. Wang, "Current and SAR induced in a human head model by the electromagnetic fields irradiated from a cellular phone," IEEE Trans. Microw. Theory Tech., vol. 42, no. 12, pp. 2249-2254, Dec. 1994.

[14] S. Gabriel, R. W. Lau, and C. Gabriel, "The dielectric properties of biological tissues: III. Parametric models for the dielectric spectrum of tissues," Phys. Med. Biol., vol. 41, no. 11, pp. 2271-2293, 1996.

[15] R. Luebbers and H. S. Langdon, "A simple model that reduces time steps needed for FDTD antenna and microstrips calculations," IEEE Trans. Antenna. Propag., vol. 44, no. 7, pp. 1000-1005, Jul 1996.

[16] G. Anzaldi, R. Santos, and F. Silva, "EMC susceptibility analysis of in-out field distribution by FDTD simulation," in FISITA 2004 Book of Abstracts Barcelona, May 2004, p. 355.

[17] G. Anzaldi, P. J. Riu, M. Fernandez, and F. Silva, "Validation procedure for final users applied to automotive environments," in Proc. Inst. Electr. Eng. Validation of Computational Electromagnetic Sem., March 2004, pp. 43-47.

[18] D. O. McCoy, D. M. Zakharia, and Q. Balzano, "Field strengths and specific absorption rates in automotive environments," IEEE Trans. Veh. Technol., vol. 48, no. 4, pp. 1287-1303, Jul 1999. 
[19] R. Mittra, "A look at some challenging problems in computational electromagnetics," IEEE Antenna. Propaga. Mag., vol. 46, no. 5, pp. 18-32, Oct. 2004.

[20] [Online]. Available: http//www.3dcafe.com

[21] K. Caputa, M. Okoniewski, and M. A. Stuchly, "An algorithm for computations of power deposition in human tissue," IEEE Antenna. Propaga. Mag., vol. 41, no. 4, pp. 102-107, Aug. 1999.

[22] J. Mix, G. Haussmann, M. Piket-May, and K. Thomas, "EMC/EMI design and analysis using FDTD," in Proc. IEEE Int. Symp. EMC, Aug. 1998, vol. 1, pp. 177-181.

[23] B. B. Beard and W. Kainz, "Review and standardization of cell phone exposure calculations using the SAM phantom and anatomically correct head models," Biomed. Eng. OnLine vol. 3, no. 34, Oct. 2004 [Online]. Available: http://www.biomedical-engineering-online.com/content $/ 3 / 1 / 34$

[24] W. Kainz, A. Christ, T. Kellom, S. Seidman, N. Nikoloski, B. Beard, and N. Custer, "Dosimetric comparison of the specific anthropomorphic mannequin (SAM) to 14 anatomical head models using a novel definition for the mobile phone positioning," Phys. Med. Biol., vol. 50, pp. 3423-3445, July 2005.

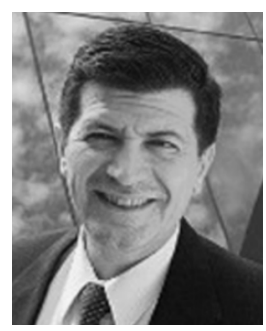

Gabriel Anzaldi was born on Buenos Aires, Argentina, on April 2, 1964. He received the M.Sc. degree in electronic engineering (with honors) from the Superior Technical School University, Buenos Aires, in 2000, and is currently working toward the Ph.D. degree in electronic technology at the Universitat Politecnica de Catalunya, Barcelona, Spain.

From 1986 to 1995, he worked for the Argentinean Government as a Telecommunication Engineer involved in the design and implementation of a wide variety of telecommunications systems. From 2000 to 2002 he was Assistant Professor at the Technical Superior School and at the National Technological University, Buenos Aires. He is currently the Technical Director at Dielro, Sant Joan Despi, Spain. His research interests include numerical electromagnetic methods in the time domain and the electromagnetic radiation effects on the human body.

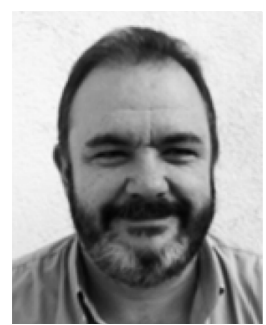

Ferran Silva (S'93-M'98) received the M.Sc. and Ph.D. degrees from the Universitat Politecnica de Catalunya (UPC), Barcelona, Spain, in 1989 and 1997, respectively.

$\mathrm{He}$ is currently Associate Professor of Electronics with the Department of Electronic Engineering, UPC. $\mathrm{He}$ is also Director of the Electromagnetic Compatibility Group in the same university (GCEM-UPC). His research interests include electromagnetic compatibility in near field and time domain with application to automotive, medical systems and installations. He has published several papers on these topics and contributed to the Wiley Encyclopedia of Biomedical Engineering (Wiley, 2006). He was a Visiting Associate Professor at the Department of Electronics, University of UK, in 2003.

Dr. Silva is currently member of the EMC Europe International Steering Committee and the chairman of the Spanish IEEE EMCS chapter.

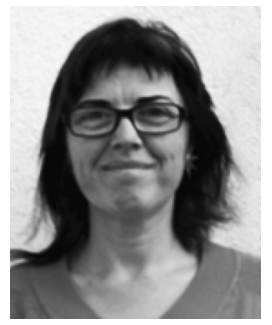

Mireya Fernández (S'91-M'97) received the M.Sc. and Ph.D. degrees from the Universitat Politecnica de Catalunya (UPC), Barcelona, Spain, in 1990 and 1996, respectively.

She is currently Associate Professor of Electronics with the Department of Electronic Engineering, UPC. She is also Quality Manager of GCEM (Electromagnetic Compatibility Group from the UPC), She was Associate Dean of the Telecommunication School (ETSETB) from the UPC (1996-2000). Her main research interests are electromagnetic compatibility in medical systems and installations, and biomedical instrumentation design focused on the design of biopotential monitoring systems. She has published several papers on these topics and contributed to the Wiley Encyclopedia of Biomedical Engineering (Wiley, 2006).

Dr. Fernández was a Visiting Researcher at the Health Sciences Department of the University of York, U.K., in 2003.

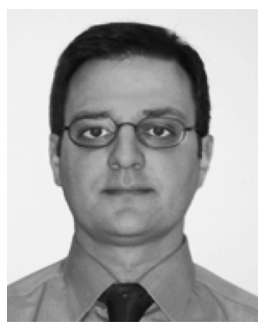

Marcos Quílez (S'97-M'05) received the M.Sc. and Ph.D. degrees from the Universitat Politecnica de Catalunya (UPC), Barcelona, Spain, in 1997 and 2004, respectively.

Since 1998, he has been with the Department of Electronic Engineering, UPC, where he is now Assistant Professor. His research interests include electromagnetic compatibility in electronic design and measurement of radiation in the near-field area.

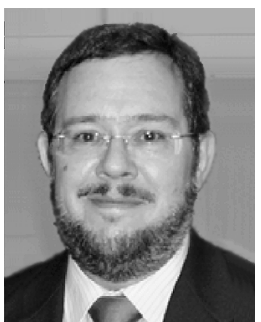

Pere J. Riu (S'86-M'91-SM'98) received the M.Sc. and $\mathrm{Ph} . \mathrm{D}$. degrees from the Universitat Politecnica de Catalunya (UPC), Barcelona, Spain, in 1986 and 1991, respectively.

$\mathrm{He}$ is currently Professor of Electronics with the Department of Electronic Engineering, UPC, where he has occupied several academic positions since 1986. His research interests include electromagnetic compatibility, computational electromagnetics, interaction of EMF with biological tissues, and biomedical instrumentation design with emphasis on electrical bioimpedance techniques, including EIT. He has published several papers on these topics and contributed to the Wiley Encyclopedia of Biomedical Engineering (Wiley, 2006). He was a Visiting Associate Professor at the Department of Bioengineering, University of Pennsylvania, PA, in 1997. Prof. Riu is currently the Interim President of the International Society for Electrical Bio-Impedance (ISEBI). 